Proceedings of the International School and Conference on Optics and Optical Materials, ISCOM07, Belgrade, Serbia, September 3-7, 2007

\title{
Electromagnetically Induced Absorption Resonance Sign Reversal
}

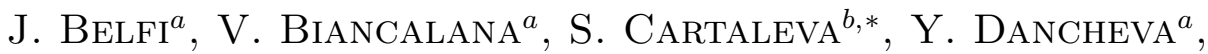 \\ E. Mariotti ${ }^{a}$, L. Moi $^{a}$, K. Nasyrov ${ }^{c}$, D. Slavov $^{b}$, \\ P. TODOROV ${ }^{b}$ AND K. VASEVA ${ }^{b}$ \\ ${ }^{a}$ CNISM-Unità di Siena, Dipartimento di Fisica Università di Siena \\ via Roma 56, 53100 Siena, Italy \\ ${ }^{b}$ Institute of Electronics, Bulgarian Academy of Sciences \\ 72 Tzarigradsko Shosse bld, 1784 Sofia, Bulgaria \\ ${ }^{c}$ Institute of Automation and Electrometry, Novosibirsk, Russia \\ Experimental results are presented about the transformation of the elec- \\ tromagnetically induced absorption resonance into the electromagnetically \\ induced transparency one. The role of the depolarization of the excited state \\ on the $D_{2}$ line of Cs atoms exposed to different confinements is discussed.
}

PACS numbers: 42.50.Gy

\section{Introduction}

The coherent population trapping (CPT) [1] and related electromagnetically induced transparency (EIT) phenomena have found a large number of applications in science and technology. The degenerate two-level systems provide many possibilities for investigation of CPT. Zeeman optical pumping destroyed by magnetic field (MF) allows the observation of EIT [2], as well as electromagnetically induced absorption (EIA) [3] in the atomic medium. CPT resonances prepared in the Hanle configuration have been investigated for atoms irradiated by monochromatic laser field in a way that different polarization components couple the Zeeman sublevels of single hyperfine (hf) ground level to a common excited state and introduce coherence between ground magnetic sublevels at zero value of a scanned around it magnetic field $B$, orthogonal to the orientation/alignment of atoms. As shown in [3], in absence of depolarizing collisions of the excited state, and depending on the ratio of the degeneracies of the two hf levels involved in the optical hf transition, EIT and EIA sub-natural width resonances can be observed for degenerate two-level systems. EIT is realized when the conditions $F_{\mathrm{g}} \rightarrow F_{\mathrm{e}}=F_{\mathrm{g}}-1, F_{\mathrm{g}}$ are met, while EIA is observed for $F_{\mathrm{g}} \rightarrow F_{\mathrm{e}}=F_{\mathrm{g}}+1$. Here, $F_{\mathrm{g}}$ and $F_{\mathrm{e}}$ are the hf quan-

*corresponding author; e-mail: stefka-c@ie.bas.bg 
tum numbers of the ground- and excited-state hf levels, respectively. However, it turns out that the EIA resonance is very sensitive to the cell buffering. It has been found [4] and recently confirmed [5] that if the cell containing alkali atoms is buffered by some noble gas, the resonance at the $F_{\mathrm{g}} \rightarrow F_{\mathrm{e}}=F_{\mathrm{g}}+1$ transitions transforms from EIA to EIT one. The resonance sign reversal has been attributed to the depolarization of the $F_{\mathrm{e}}$ level by collisions of alkali atoms with the buffer gas atoms. At the same time these collisions do not lead to the depolarization of the $F_{\mathrm{g}}$ level, thus preserving the coherent superposition of the ground-state magnetic sublevels introduced by the light at $B=0$. Let us note that the cell buffering does not reverse the sign of the EIT resonances observed on $F_{\mathrm{g}} \rightarrow F_{\mathrm{e}}=F_{\mathrm{g}}-1$, $F_{\text {g }}$ transitions.

It has been recently shown [6] that the use of extremely thin cell (ETC, [7]) of thickness $L$ equal to the light wavelength $\lambda$ allows one to examine the CPT on each individual hf transition of $\mathrm{Cs} D_{2}$ line. In this way processes resulting in EIT or EIA resonance formation can be distinguished (Fig. 1). For cm size cells, this cannot be accomplished due to the complete Doppler overlapping of hf transitions. Moreover, ETC gives the possibility to separate the contribution of slow and fast atoms to the CPT signal. The CPT signal in ETC has been observed [6] in transmission but only for "slow" atoms, i.e. mainly for atoms flying approximately parallel to the cell windows. In addition, the EIA resonance sign reversal has been reported for $F_{\mathrm{g}} \rightarrow F_{\mathrm{e}}=F_{\mathrm{g}}+1$ transitions. A theoretical model has been proposed based on the optical Bloch equations that involves the elastic interaction processes of atoms confined in the ETC with its walls resulting in depolarization of the Cs excited state. This depolarization leads to the sign reversal of the EIA resonance.

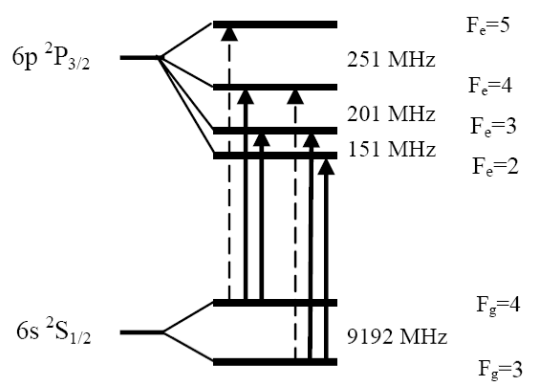

Fig. 1. Optical hf transitions on the $D_{2}$ line of Cs: $F_{\mathrm{g}} \rightarrow F_{\mathrm{e}}=F_{\mathrm{g}}-1, F_{\mathrm{g}}$ transitions (solid line); $F_{\mathrm{g}} \rightarrow F_{\mathrm{e}}=F_{\mathrm{g}}+1$ (dashed line).

In this communication we present the first experimental observation of the EIA resonance sign reversal in cm size, non-buffered cell. In this particular case, the cell walls are coated by anti-relaxation coating which preserves the ground-state spin orientation even after a great number of atom-wall collisions. To start 
a systematic study of the EIA resonance sign reversal that is observed on the $D_{2}$ line of $\mathrm{Cs}$, the CPT resonances are registered in $\mathrm{cm}$ size cell (with and without anti-relaxation coating), cm size, buffered by 2 Torr Ar cell and ETC, under similar experimental conditions.

\section{Experimental results and discussion}

We used (Fig. 2) an extended cavity diode laser (ECDL), operating in single-frequency mode at $\lambda=852 \mathrm{~nm}$ and with $3 \mathrm{MHz}$ line width. The laser beam was directed at several types of cells: cm size glass cell containing pure Cs; buffered cm size cell — with 2 Torr of Ar added; cm size cell with pure Cs and anti-relaxation, poly-dimethyl-siloxane (PDMS) coating of the walls [8]; and ETC. All cells were placed between a pair of Helmholtz coils providing variable strengths $\mathrm{MF} B$ in an orthogonal to the atomic orientation/alignment direction (along $y$ axis in Fig. 2). No shielding against laboratory MF $B_{\text {str }}$ is provided. In case of $\mathrm{cm}$ size cells (kept

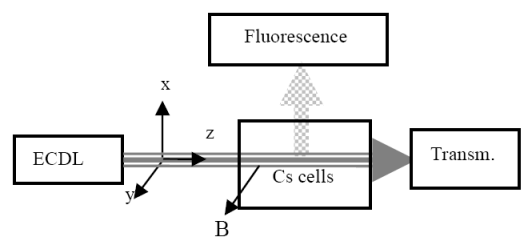

Fig. 2. Scheme of experiment.

at $20^{\circ} \mathrm{C}$ ), experiments were performed irradiating Cs atoms by circularly polarized light, while for the ETC (Cs source at $110^{\circ} \mathrm{C}$ ) linearly polarized (along $x$ axis) light is used. The EIT/EIA resonances are observed in transmission and in fluorescence as a function of MF $B$ which is modulated around $B=0$.

2.1. EIA resonances in the fluorescence and absorption, observed in non-coated $\mathrm{cm}$ size cell containing pure Cs atoms

In $\mathrm{cm}$ size cell, the profiles of the three hf transitions starting from a single $F_{\mathrm{g}}$ level strongly overlap forming a single absorption (fluorescence) line where the three hf transitions (Fig. 1) responsible for the formation of both EIT and EIA resonances are involved. When exciting atoms by single-mode laser light and performing the experiment in dilute Cs vapor (thus avoiding velocity changing collisions between Cs atoms), the three hf transitions forming the absorption line will be independently excited, each one at different velocity class of atoms. Under these conditions the open transitions are depleted because of the hf optical pumping to the ground hf level that does not interact with the laser light. Therefore, they do not play a significant role in the formation of the resonance. At the same time, the closed transition that does not suffer hf optical pumping mainly determines the sign of the resonance. Thus, as shown in [4] the EIA resonance is observed for the absorption line starting from the $F_{\mathrm{g}}=4$ level. Our experiment confirms (Fig. 3) 


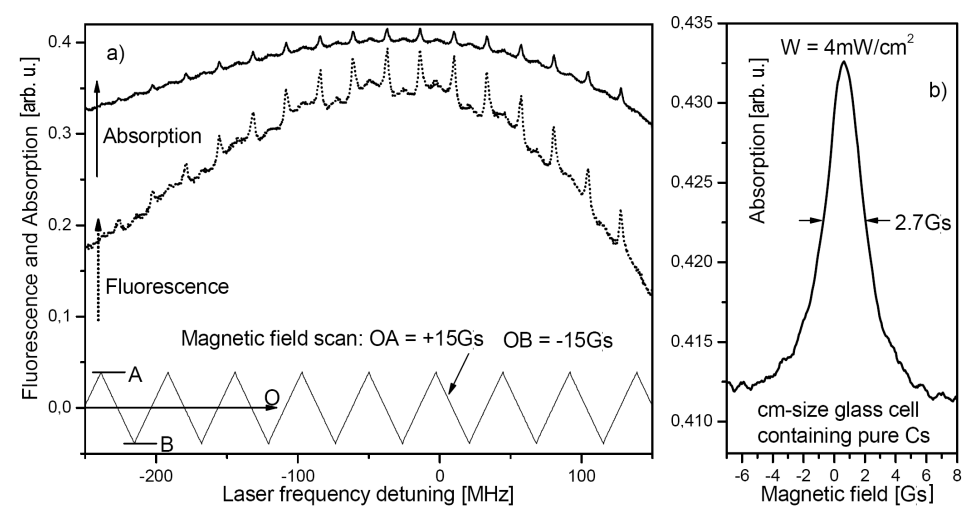

Fig. 3. (a) EIA resonances in fluorescence and absorption during the double scan of laser frequency and MF (frequency detuning: from $F_{\mathrm{g}}=4 \rightarrow F_{\mathrm{e}}=5$ transition); (b) an example of the EIA resonance in absorption.

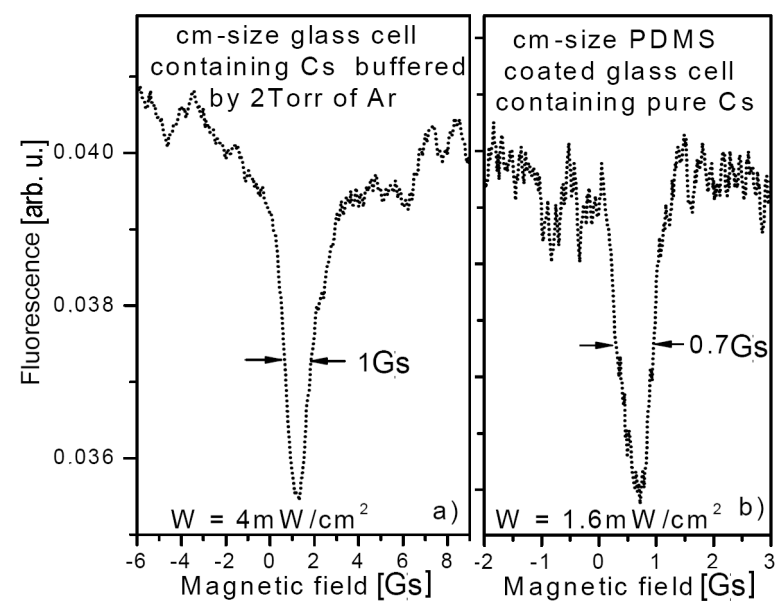

Fig. 4. EIA resonance sign reversal (see also Fig. 3) in buffered (a) and coated (b) cells.

the previous results showing the EIA resonance formation in a large frequency region around the $F_{\mathrm{g}}=4 \rightarrow F_{\mathrm{e}}=5$ transition. The approach of the double scan is performed [4]: the laser frequency is slowly scanned over the absorption line while the $\mathrm{MF}$ is scanned around $B=0$ with a higher frequency. Such an experiment makes possible the observation of a sequence of resonances, superimposed on the absorption/fluorescence profile. The maxima of the EIA resonances are always observed at $B=0$.

\subsection{EIA resonance sign reversal in buffered $\mathrm{cm}$ size cell and anti-relaxation coated non-buffered $\mathrm{cm}$ size cell}

Our experiment performed with cm size Cs cell buffered by 2 Torr of Ar also confirms the previous results [4]. If the cell containing alkali atoms is buffered 
by noble gas, the resonance at the $F_{\mathrm{g}}=4 \rightarrow F_{\mathrm{e}}=5$ transition is changed from EIA to EIT one. Namely, only the EIT resonance is observed at the $F_{\mathrm{g}}=4$ set of transitions (illustrated in Fig. 4a). The transformation of the resonance sign is attributed to the depolarization of the $F_{\mathrm{e}}$ level by Cs atoms-buffer gas atoms collisions. At the same time, these collisions do not lead to depolarization of the $F_{\text {g level. }}$

The main goal of the presented work is to examine the consequences of Cs atoms - PDMS coated wall collisions in the $\mathrm{cm}$ size cell. From the numerous optical pumping and CPT experiments, it is well known that such collisions do not cause the spin reorientation at the $F_{\mathrm{g}}$ hf levels, even after many thousands of events. The idea of our experiment (based on the sensitivity of the EIA resonance to the depolarization of the excited state) is to test if the interaction of Cs atoms with the cell wall coating during the collision will be sufficient for the depolarization of the excited state. Our experiments performed with a $\mathrm{cm}$ size cell containing pure Cs vapour (its walls coated by the PDMS) have shown that the EIA resonance is transformed into EIT one when observed on the $F_{\mathrm{g}}=4$ set of transitions. This first experimental evidence of the EIA resonance sign reversal in coated cell (Fig. 4b) might be considered as a support to the statement that the weak atom-coated wall interaction is sufficient to depolarize the excited state. Further work is in progress for clarification of different processes contributing to the EIA resonance sign reversal in coated cells.

\subsection{EIA resonance sign reversal in non-buffered ETC cell}

As discussed in the Introduction the absorption and fluorescence spectra in ETC differ significantly from those observed in cm size cells (see [9] and references therein). Here we illustrate (Fig. 5a) only the case of $L=\lambda$. Under this condition narrow dips of reduced absorption occur in the absorption spectrum, centered at each hf transition, i.e. "slow" atoms are mainly responsible for the formation of

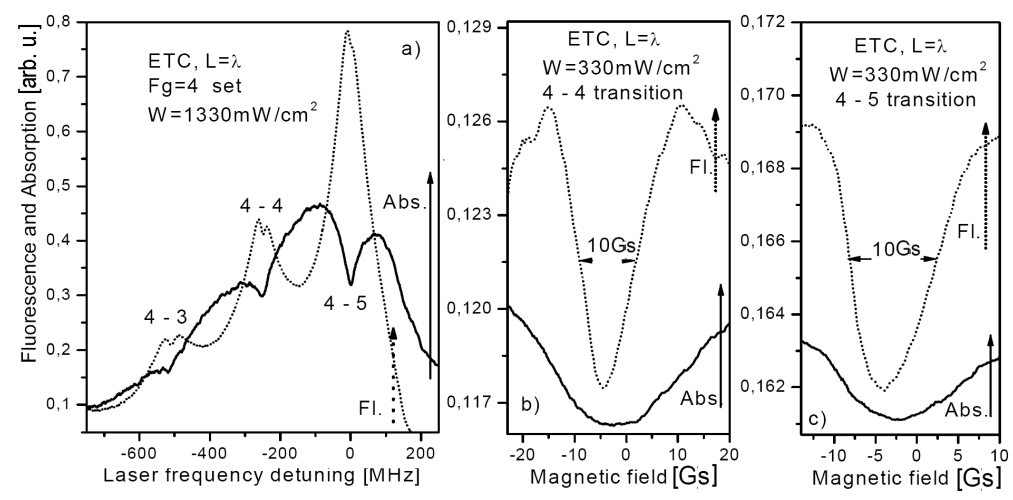

Fig. 5. (a) Fluorescence and absorption spectra of ETC with $L=\lambda$, at $F_{\mathrm{g}}=4$ set of hf transitions; (b) EIT resonance at $F_{\mathrm{g}}=4 \rightarrow F_{\mathrm{e}}=4$ transition; (c) illustration of the EIA resonance sign reversal at $F_{\mathrm{g}}=4 \rightarrow F_{\mathrm{e}}=5$ transition. 
these features. The observed dips are attributed to velocity-selective saturation of the $\mathrm{hf}$ transition and to velocity-selective population loss due to optical pumping to the ground level that does not interact with the light field. In fact, to complete saturation and/or optical pumping, the atom needs a longer interaction time with the light than for a single event of absorption. The "slow" atoms are also responsible for the significant narrowing of the fluorescence profiles of different hf transitions which make possible their separation in the ETC (Fig. 5a). As shown in [6] these atoms contribute to the coherent resonance formation. Here we present the new results related to the EIT resonances observed in the fluorescence of the ETC (Fig. 5b,c) which are almost one order of magnitude narrower than EIT resonances in the absorption (in Fig. 5b,c only the bottom of the EIT resonance in absorption is shown). Also the EIA resonance at the $F_{\mathrm{g}}=4 \rightarrow F_{\mathrm{e}}=5$ fluorescence is transformed into the EIT one when observed in the ETC (Fig. 5c). This transformation is attributed to the Cs excited state depolarization by the ETC window influence.

The presented results are promising for new applications of the EIA resonances in the field of collision and atom-surface interaction studies.

\section{Acknowledgments}

Authors appreciate the supports from INTAS project (grant: 06-1000017-9001), CNR-BAS collaboration, B.F.S.R. project (grant: F-1404/04) and RFBR project (grant: 07-02-00954).

\section{References}

[1] G. Alzetta, A. Gozzini, L. Moi, G. Orriols, Nuovo Cimento 36, 5 (1976); E. Arimondo, Prog. Opt. 35, 257 (1996).

[2] F. Renzoni, W. Maichen, L. Windholz, E. Arimondo, Phys. Rev. A 55, 3710 (1997).

[3] Y. Dancheva, G. Alzetta, S. Cartaleva, M. Taslakov, Ch. Andreeva, Opt. Commun. 178, 103 (2000); F. Renzoni, C. Zimmermann, P. Verkerk, E. Arimondo, J. Opt. B, Quantum Semiclass. Opt. 3, S7 (2001).

[4] C. Andreeva, S. Cartaleva, Y. Dancheva, V. Biancalana, A. Burchianti, C. Marinelli, E. Mariotti, L. Moi, K. Nasyrov, Phys. Rev. A 66, 012502 (2002).

[5] D.V. Brazhnikov, A.M. Tumaikin, V.I. Yudin, A.V.Taichenachev, J. Opt. Soc. Am. B 22, 57 (2005); D.V. Brazhnikov, A.V. Taichenachev, A.M. Tumaikin, V.I. Yudin, S.A. Zibrov, Ya.O. Dudin, V.V. Vasil'ev, V.L. Velichansky, JETP Lett. 83, 64 (2006).

[6] C. Andreeva, A. Atvars, M. Auzinsh, K. Bluss, S. Cartaleva, L. Petrov, D. Slavov, http://arxiv.org/abs/0704.3010.

[7] D. Sarkisyan, D. Bloch, A. Papoyan, M. Ducloy, Opt. Commun. 200, 201 (2001).

[8] M. Allegrini, P. Bicchi, L. Moi, P. Savino, Opt. Commun. 32, 396 (1980).

[9] C. Andreeva, S. Cartaleva, L. Petrov, S.M. Saltiel, D. Sarkisyan, T. Varzhapetyan, D. Bloch, M. Ducloy, Phys. Rev. A 76, 013837 (2007). 